\title{
Photo-induced changes in the Langmuir adsorption constants of metal oxide layers in self- cleaning cation sensors.
}

\author{
Philip S. Foran ${ }^{1}$ and Colin Boxall ${ }^{1}$ \\ ${ }^{1}$ Engineering Department, Lancaster University, Lancaster, LA1 4YW, UK.
}

\begin{abstract}
For the first time, we have used a metal oxide-coated quartz crystal microbalance (QCM) to measure $\mathrm{Cs}^{+}$adsorption onto illuminated and un-illuminated mesoporous $\mathrm{TiO}_{2}\left(\mathrm{~m}-\mathrm{TiO}_{2}\right)$ films by microgravimetric means in-situ. In the simplest case, such experiments yield two parameters of interest: $\mathrm{K}$, the Langmuir adsorption coefficient and $m_{\max }$ the maximum mass of adsorbate to form a complete monolayer at the $\mathrm{m}-\mathrm{TiO}_{2}$-coated quartz crystal piezoelectric surface. Importantly, we have found that illumination of the $\mathrm{m}-\mathrm{TiO}_{2}$ film with ultra bandgap light results in an increase in $m_{\max }$ i.e. illumination allows for greater adsorption of substrate to occur than in the dark. Our studies also show that under illumination, $\mathrm{K}$ also increases indicating a higher affinity for surface adsorption. The photoinduced change in $m_{\max }$ and $\mathrm{K}$ are thought to be due to an increase in surface bound titanol groups, thus increasing the number of available adsorption sites - and so providing evidence to support the notion of photoinduced adsorption processes in photocatalytic systems. These findings have implications for the development of a reversible adsorption based microgravimetric sensor for $\mathrm{Cs}^{+}$.
\end{abstract}

\section{INTRODUCTION}

Functional metal oxides such as $\mathrm{TiO}_{2}$ find use as, inter alia, anode materials in dye sensitised solar cells, photocatalysts for gas / liquid phase pollutant destruction and self-cleaning glass coatings [1]. In particular, the latter two applications rely upon the photo-generation of energetic charge carriers within the $\mathrm{TiO}_{2}$ matrix to drive the oxidation of organic contaminants or putative surface reconstruction resulting in an increase in surface bound titanol groups. The latter may increase both surface wettability and the number of surface sites at which pollutant substrate adsorption may occur prior to their destruction at that surface. Whether such photoinduced adsorption effects exist is one of the unsolved questions of photocatalysis. In 2003, Shultz et al. used sum frequency generation, a highly surface sensitive technique, to analyse $\mathrm{TiO}_{2}$ films under illumination and for the first time measured an increase in the surface hydroxyl count, which would increase the surface wettability. Their results also detected trace amounts of organics on the surface of as-prepared $\mathrm{TiO}_{2}$ coatings in ambient air. However, after UV irradiation, these trace organics were no longer present, therefore exposing more of the $\mathrm{TiO}_{2}$ surface and potentially further increasing the surface wettability [2].

The Quartz Crystal Microbalance (QCM) is a stable, extremely sensitive device capable of detecting mass changes of a few nanograms (ng) per square centimetre [3], such as those that may accompany photo-induced changes in substrate adsorption at $\mathrm{TiO}_{2}$ surfaces. The quantitative relationship between the change in frequency, $\Delta \mathrm{f}(\mathrm{Hz})$, of a piezoelectric crystal and the mass change caused by mass loading, $\Delta \mathrm{m}(\mathrm{g})$ on the piezoelectric crystal surface was firstly derived by Sauerbrey, and is given by [ Eq. 1] where $\mathrm{C}_{\mathrm{f}}$ is a constant of linear proportionality whose value may be derived by calibration or by theoretical calculation: 


$$
\Delta f=-C_{f} \Delta m
$$

[ Eq. 1]

The QCM has been employed to determine Langmuir parameters $\mathrm{K}$, the Langmuir adsorption coefficient and $m_{\max }$, the mass of a complete monolayer of adsorbate, for organic pesticides adsorbing at illuminated \& un-illuminated $\mathrm{TiO}_{2}$ coated quartz crystal surfaces [4]. However, in these experiments the organic adsorbent was destroyed at the illuminated $\mathrm{TiO}_{2}$ surface via photo-oxidation and therefore gave lower than expected values for K. Thus, in order to deconvolute photoinduced changes in Langmuir behaviour on $\mathrm{TiO}_{2}$ due to substrate decomposition and/or changes in the nature of the adsorbing surface (photoinduced adsorption), an adsorbate that is not degradable by photocatalysis must be used. This adsorbate must also couple with the resonator to have an effect on mass and therefore give measurable change in resonant frequency. Of interest here are the electrochemically inert alkali \& alkaline earth metal cations, especially the heavier members of these groups, Cs \& Sr.

${ }^{137} \mathrm{Cs}$ and ${ }^{90} \mathrm{Sr}$ are radionuclides resulting from nuclear fission. They are highly radiotoxic and have a half-life of about 30 years, making them a substantial hazard should they be released into the environment, from, for example, stored spent nuclear fuel pins in interim storage or in final geological repository. Current detection methods for ${ }^{137} \mathrm{Cs}$ and ${ }^{90} \mathrm{Sr}$ include ICP-MS (nonradiometric) and scintillation detectors (radiometric), neither of which are well suited to in-situ detection in aqueous streams. $\mathrm{Cs}^{+}$ions are not degraded via photocatalysis therefore providing a means to (i) study the Langmuir adsorption parameters $\mathrm{K}$ and $m_{\max }$ on $\mathrm{TiO}_{2}$ films in both the dark and under band-gap illumination, so investigating the photoinduced adsorption processes; (ii) explore the potential implications on this effect in the development of a reversible, in-situ, adsorption based microgravimetric sensor for $\mathrm{Cs}^{+}$.

\section{EXPERIMENTAL}

$5 \mathrm{MHz}$ AT-cut QCM crystals (Inficon, UK) were coated with a mesoporous- $\mathrm{TiO}_{2}$ (m$\mathrm{TiO}_{2}$ ) layer via sol-gel spin coating as follows. A reverse micellar sol-gel was prepared, as described by Yu et al. [5]. The resultant sol-gel was applied to quartz piezoelectrode substrates by spin coating for $10 \mathrm{~s}$ at $2900 \mathrm{rpm}$. Coated substrates were then fired in a furnace at $350{ }^{\circ} \mathrm{C}$ for $1 \mathrm{~h}$ to anneal the $\mathrm{TiO}_{2}$ and produce a resilient coating. Annealing at this temperature has been shown to produce $\mathrm{TiO}_{2}$ with predominantly the anatase structure by Raman analysis (not shown) [6]. QCM experiments were performed using a Maxtek research quartz crystal microbalance (RQCM) and data recorded on a desktop PC using the Maxtek RQCM logging software at a rate of 1200 data points per minute. Frequency responses are initially measured in $\mathrm{Hz}$, to convert this to meaningful mass in ng a Sauerbrey sensitivity factor of $\mathrm{C}_{\mathrm{f}}=6.217 \mathrm{ng} / \mathrm{Hz}$ was used [7], this allows for the calculation of adsorbed $\mathrm{Cs}^{+}$concentration.

Illumination with $315 \mathrm{~nm}$ monochromated light was provided by a $900 \mathrm{~W}$ xenon lamp (Applied Photophysics) monochromation also served to reduce heating of the gold substrate. The semiconductor-coated quartz crystal oscillator was placed in a borosilicate beaker with a dedicated flat quartz window to minimise any scattering of and absorption of the incident light. The beaker was submerged in a thermostatic water-bath set at $30^{\circ} \mathrm{C}$ a close match for the crystal's turn-around point, minimising the effect of temperature fluctuations on the recorded frequency. This could not be completely eliminated, and noise with amplitude of about $0.5 \mathrm{~Hz}$ is noticeable. 
All reagents, including $\mathrm{CsNO}_{3}(>99 \%$, purum p.a., Fluka, UK) were obtained from reputable suppliers at the highest available purity and used as received. Solutions of $\mathrm{CsNO}_{3}$ were prepared immediately prior to the start of each experiment. The solutions were prepared using doubly deionized water (Millipore Milli-Q system) to a resistivity of $1.8 \times 10^{5} \Omega \cdot \mathrm{m}$. Solutions were deoxygenated by bubbling with nitrogen (Oxygen-Free, BOC, UK) prior to experiments at a higher relative flow rate and during experiments at a significantly lower flow rate through a filtered glass Pasteur pipette placed behind the crystal holder to minimize potential disturbance at the crystal face. This slow bubbling of $\mathrm{N}_{2}$ also aided in solution mixing, during any one QCM run, experiments were calibrated in-situ until a stable baseline frequency was achieved. All experiments were performed in a dedicated dark room to eliminate the effects of ambient light on the system.

\section{DISCUSSION}

\section{$\underline{\text { Under Dark Conditions }}$}

Frequency response of the $\mathrm{m}-\mathrm{TiO}_{2}$ coated crystal as a function of time in dark conditions at increasing $\mathrm{Cs}^{+}$concentrations is shown in Figure 1a. Concentration of $\mathrm{Cs}^{+}$at the start of the experiment was $0 \mathrm{mM}$. The $\mathrm{Cs}^{+}$concentration in the test solution was increased to $0.25 \mathrm{mM}$ by addition of an aliquot from a caesium nitrate stock solution (0.01M) after 60 minutes equilibration and resulted in a rapid frequency decrease of $14 \mathrm{~Hz}$. Increasing $\mathrm{Cs}^{+}$concentration in the solution induces a decrease in frequency at the QCM surface as a result of adsorption of $\mathrm{Cs}^{+}$ onto the $\mathrm{m}$-TiO2 matrix. Subsequent additions of $\mathrm{Cs}^{+}$at $60 \mathrm{mins}$ intervals increased the concentration to $0.5 \mathrm{mM}, 0.75 \mathrm{mM}$ and $1 \mathrm{mM}$ with a measured frequency response of $-18 \mathrm{~Hz}$, $19 \mathrm{~Hz}$ and $-19 \mathrm{~Hz}$, respectively.

The Langmuir isotherm is defined by [ Eq. 2]:

$$
m=\frac{m_{\max } K C}{1+K C}
$$

where $m$ is the concentration of mass adsorbed $(\mathrm{mM}), \mathrm{C}$ is the concentration in solution $(\mathrm{mM})$ and $\mathrm{K}$ is the Langmuir adsorption coefficient and $m_{\max }$ is the maximum mass of adorbate (in this case $\mathrm{Cs}^{+}$) to form a complete monolayer at the $\mathrm{m}-\mathrm{TiO}_{2}$-coated quartz crystal surface. $m$ is determined experimentally (directly from QCM experiments in this case) at different values of $\mathrm{C}$ and a plot of $1 / m v s .1 / \mathrm{C}$, in accordance with the reciprocal form of the Langmuir isotherm [Eq. $3 \mathrm{a}$ ], yields a straight line where the gradient and intercept can be used to evaluate $\mathrm{K}$ and $m_{\max }$.

$$
\frac{1}{m}=\frac{1}{K m_{\max }}\left(\frac{1}{C}\right)+\frac{1}{m_{\max }} \quad \frac{m_{\max }}{m}=\frac{1}{K}\left(\frac{1}{C}\right)+1
$$

Thus, by using [Eq. 1], the frequency changes of Figure 1a may be converted into mass changes and used in a plot of $m_{\max } / m$ vs. 1/C, which yields values of $\mathrm{K}$ and $m_{\max }$ of 6.7 and 22.5

respectively, Figure $1 \mathrm{~b}$. 

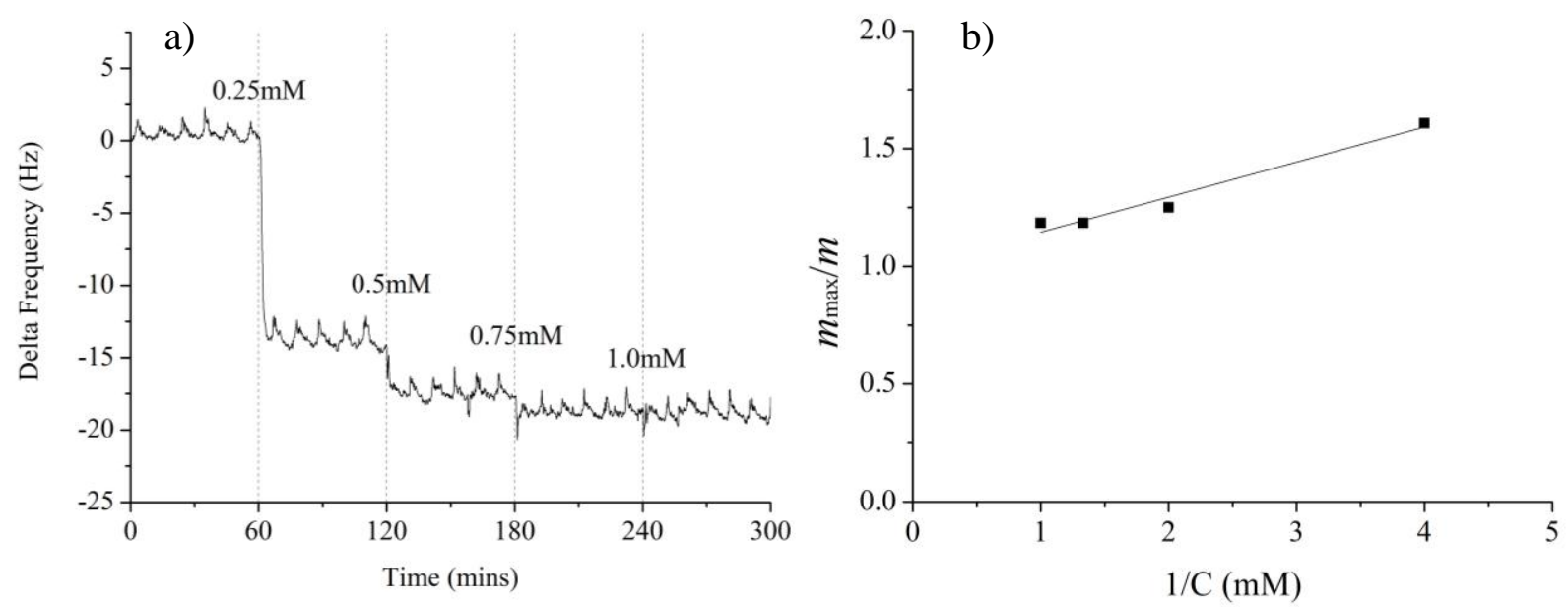

Figure 1. a) Frequency response of the $\mathrm{m}-\mathrm{TiO}_{2}$ crystal at different concentrations of $\mathrm{Cs}^{+}$under dark conditions. At $\mathrm{t}=0 \mathrm{mins} \mathrm{C}=0 \mathrm{mM}$. Concentration was increased by $250 \mu \mathrm{M}$ at 60 minute intervals. b) Langmuir isotherm plot - response of the $\mathrm{m}-\mathrm{TiO}_{2}$ crystal in dark conditions to $\mathrm{Cs}^{+}$ concentration. Gradient $=0.1486$, intercept $=0.9967$.

\section{$\underline{\text { Under UV Illumination }}$}

The frequency response of the $\mathrm{m}-\mathrm{TiO}_{2}$ coated QCM under illumination was investigated using a different experimental protocol to the dark response as follows. The coated crystals were mounted in its QCM holder and exposed to $\mathrm{Cs}^{+}$containing solutions $(0,0.05,0.1$ and $0.2 \mathrm{mM})$. The QCM's signal was allowed to stabilise in the dark in the test solution for $24 \mathrm{hrs}$ prior to illumination until no drift in frequency was observed. The recorded $f v s$. time data of Figure $2 \mathrm{a}$ shows the last hour of stabilisation of the QCM in the dark, with illumination at $315 \mathrm{~nm}$ starting at time $=60$ minutes.

As reported by Lemon \& Hupp [8], the rise in frequency, is due to local heating of the gold electrode on the crystal and not due to an apparent mass loss. This thermally induced frequency rise can be compensated for by performing the experiment in the absence of $\mathrm{Cs}^{+}$and using the resultant $f$ vs. time trace as a baseline reference. Therefore, the frequency responses for $0.05 \mathrm{mM}, 0.1 \mathrm{mM}$ and $0.2 \mathrm{mM}$ were $-20 \mathrm{~Hz},-29 \mathrm{~Hz}$ and $-35 \mathrm{~Hz}$ respectively.

To exclude the possibility of the frequency rise/apparent mass loss being due to the photo-oxidation of surface contaminants, experiments were performed in a randomised order of $0 \mathrm{mM}, 0.1 \mathrm{mM}, 0.2 \mathrm{mM}$ and finally $0.05 \mathrm{mM}$. Performing experiments from the lowest concentration incrementally would decrease the amount of surface organics available for photooxidation after each illumination period, resulting in a smaller frequency rise causing the incorrect interpretation of mass gain or $\mathrm{Cs}^{+}$adsorption. However, performing the experiments in the aforementioned order overcomes this issue. 

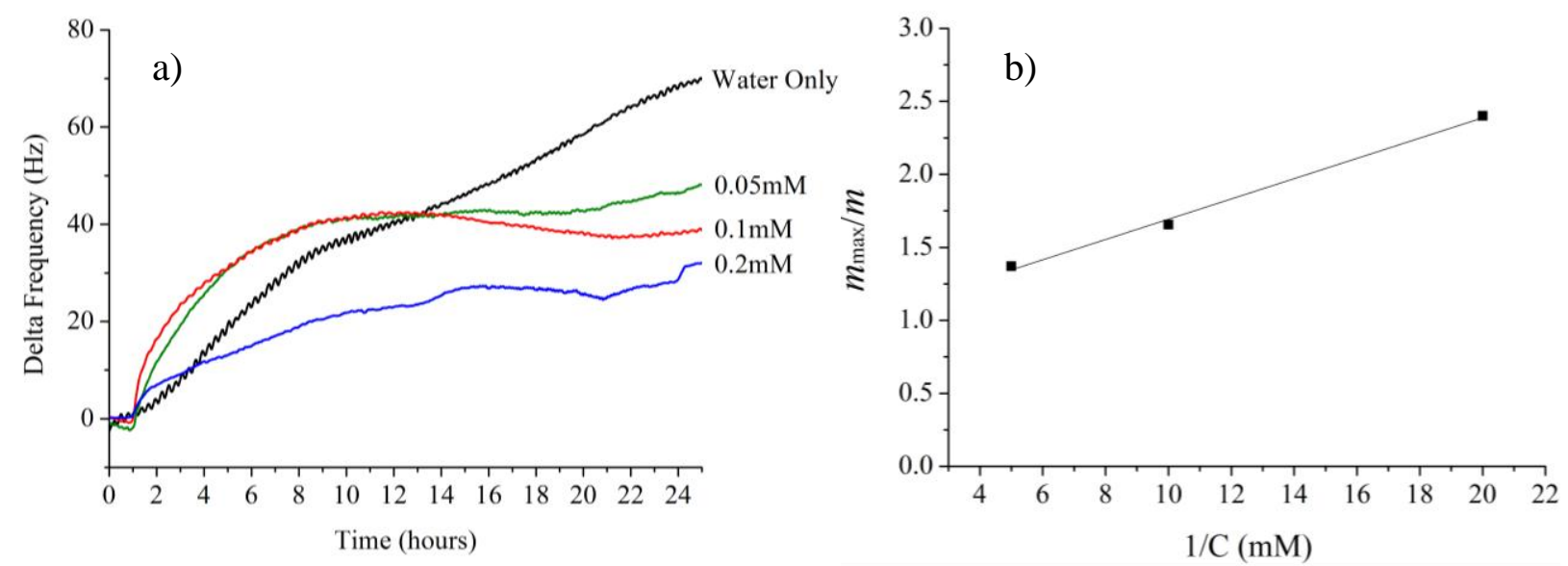

Figure 2. a) Frequency response of the $\mathrm{m}-\mathrm{TiO}_{2}$ crystal at different concentrations of $\mathrm{Cs}^{+}$under illumination. $\mathrm{C}=0 \mathrm{mM}, 0.05 \mathrm{mM}, 0.1 \mathrm{mM}$ and $0.2 \mathrm{mM}$. b) Langmuir isotherm plot - response of the $\mathrm{m}-\mathrm{TiO}_{2}$ crystal under illumination to $\mathrm{Cs}^{+}$concentration. Gradient $=0.0694$, intercept $=0.999$.

As in Figure 2a, mass change response for each added $\mathrm{Cs}^{+}$concentration may be determined using [ Eq. 1] and used in a plot of $m_{\max } / m v s .1 / \mathrm{C}$, which yields values of $\mathrm{K}$ and $m_{\max }$ of 14.4 and 48 respectively, Figure $2 b$.

It can be seen that under illumination the Langmuir adsorption coefficient, $\mathrm{K}$, approximately doubles to the value of 14.4 from the value $\mathrm{K}=6.7$ obtained in the dark. Similarly, $m_{\max }$ approximately doubles to a value of 48 when the $\mathrm{m}-\mathrm{TiO}_{2}$ coated crystal is illuminated compared to a value of 22.5 obtained in the dark, indicating that illumination allows for greater adsorption of $\mathrm{Cs}^{+}$to occur on the $\mathrm{TiO}_{2}$ surface than in the dark. Two mechanisms can be envisaged: First, an intercalation of $\mathrm{Cs}^{+}$cations with the $\mathrm{TiO}_{2}$ film to compensate a photolytically induced charge accumulation layer on the $\mathrm{TiO}_{2}$ surface as described by Lemon and Hupp [8], However, no hole $\left(\mathrm{h}^{+}\right)$scavengers are present in this system to prevent recombination of photo-excited electrons, and therefore promote the build-up of such a charge layer, rendering this mechanism unlikely. Second, the increased adsorption is due to the result of an increase in surface titanol concentration in a manner analogous to that thought to give rise to photo-induced super-hydrophilic $\mathrm{TiO}_{2}$ surfaces, therefore increasing available surface sites for $\mathrm{Cs}^{+}$adsorption.

The detection of $\mathrm{Cs}^{+}$ions in both dark conditions and under illumination on $\mathrm{TiO}_{2}$ films provides a means to micro gravimetrically measure $\mathrm{Cs}^{+}$ions in solution. This may be adapted for use in ground water situations for the detection of ${ }^{137} \mathrm{Cs}^{+}$in-situ as a replacement for external analysis methods currently employed such as ICP-MS. However, aqueous sensors are prone to bio-fouling over time causing the sensors to eventually cease to function [9]. The photo-active properties of the metal-oxide $\mathrm{m}-\mathrm{TiO}_{2}$ films used in this study may be exploited to enhance sensor performance two-fold. Firstly by photo-oxidation of surface organic contaminants therefore cleaning the sensor surface and preventing build-up of said bio-films and reducing the amount of maintenance required, which is especially desirable in a nuclear environment. Secondly, the sensitivity of the sensor may be improved by increasing the number of available adsorption sites for $\mathrm{Cs}^{+}$ions via photo-condensation. Adsorption based sensors also suffer from a lack of an easy method of analyte desorption, this is typically achieved using a solvent wash. However, the photo-induced adsorption on metal-oxide films may be a switchable process. Whether $\mathrm{Cs}^{+}$is expelled upon the removal of illumination is currently under investigation. 


\section{CONCLUSIONS}

We have deposited mesoporous- $\mathrm{TiO}_{2}$ films onto QCM crystals and successfully detected $\mathrm{Cs}^{+}$in solution at various concentrations, with potential applications as a caesium sensor. Using this device we have shown that, compared to the dark, $\mathrm{TiO}_{2}$ films exhibit a change in Langmuir constants, $\mathrm{K}$ and $m_{\max }$ of $\mathrm{Cs}^{+}$adsorption when illuminated. Specifically, we have found that illumination of the $\mathrm{m}-\mathrm{TiO}_{2}$ film results in $m_{\max }$ approximately doubling i.e. illumination allows for greater adsorption of $\mathrm{Cs}^{+}$to occur than in the dark. Our studies also show that $\mathrm{K}$ approximately doubles, indicating a higher affinity for surface adsorption under illumination, providing support for the surface reconstruction based explanation (increase in surface titanol group concentration) of photo-induced super-hydrophilicicity mechanism on metal oxide films.

\section{ACKNOWLEDGMENTS}

The authors wish to thank the Engineering and Physical Science Research Council UK for provision of a CASE award to support PSF, and Oxley Developments Ltd and The Lloyds Register Educational Trust for additional financial support. The Lloyds Register Educational Trust is an independent charity working to achieve advances in transportation, science, engineering and technology education, training and research worldwide for the benefit of all.

\section{REFERENCES}

[1] A. Fujishima, X. Zhang and D. A. Tryk, Surface Science Reports 63 (12), 515-582 (2008).

[2] C.-y. Wang, H. Groenzin and M. J. Shultz, Langmuir 19 (18), 7330-7334 (2003).

[3] F. Pascal-Delannoy, B. Sorli and A. Boyer, Sensors and Actuators a-Physical 84 (3), 285291 (2000).

[4] C. Boxall and M. Muneer, presented at the Third International Conference on Semiconductor Photochemistry, University of Strathclyde, Glasgow, Scotland, 2010 (unpublished).

[5] J. C. Yu, J. G. Yu, W. K. Ho and J. C. Zhao, Journal of Photochemistry and Photobiology a-Chemistry 148 (1-3), 331-339 (2002).

[6] X. D. Wang, J. Shen and Q. Pan, J. Raman Spectrosc. 42 (7), 1578-1582 (2011).

[7] P. Foran and C. Boxall, Unpublished Work.

[8] B. I. Lemon and J. T. Hupp, The Journal of Physical Chemistry 100 (35), 14578-14580 (1996).

[9] H. Ben-Yoav, T. Amzel, A. Biran, M. Sternheim, S. Belkin, A. Freeman and Y. Shacham-Diamand, Sensors and Actuators B: Chemical 158 (1), 366-371 (2011). 